binding in the membraneous fraction of cells, may provide an alternative site for colchicine inhibition. Substantial amounts of tubulin are found in the post-synaptic functional lattice (Walters et al., Nature, 257, 496; 1975) and in synaptic membranes and vesicles (Blitz et al., Proc. natn. Acad. Sci. U.S.A., 71, 4144; 1974). There is little doubt that tubulin is present in these membrane preparations, as the protein was carefully characterised by SDS gel electrophoresis and peptide mapping. The main difficulty is to be sure that the soluble cytoplasmic tubulin is not trapped or adsorbed to the membrane during preparation.

Using thyroid and neuronal membranes, Bhattacharyya et al. (J. biol. Chem., 250, 7639; 1975) attempted to resolve this problem. By adding radioactive tubulin the contamination of these membranes by cytoplasmic tubulin was estimated as $0.1 \%$. Nevertheless, without knowing the relative specific activities of the membrane and soluble tubulin, it is still possible that this small contamination represents a significant proportion of the membrane protein. Another problem is to consider what radioactive tubulin to add, as a label for soluble tubulin. 6S tubulin may not equilibrate with the ring form (36S) of tubulin or fragments of microtubules (Weingarten et al., Proc. natn. Acad. Sci. U.S.A., 72, $1858 ; 1975)$.

In these circumstances a better control may be to add radioactive microtubules. Another method of assaying the putative tubulin is by colchicine binding. In thyroid and neuronal membranes prepared by two different methods, colchicine binding activity is present far in excess of contaminating soluble tubulin. So the latter may not be such a problem. Colchicine binding to membranes by itself is not surprising since the molecule is so hydrophobic. What suggests that this binding site is indeed tubulin, is its respective affinities for colchicine and its analogues, and vinblastine, which are identical to those of soluble tubulin. Furthermore, the colchicine binding site can be solubilised with an enrichment in both its activity and in a protein co-migrating with cytoplasmic tubulin on SDS gel electrophoresis. The membrane colchicine site is more heat stable and has a higher optimum binding temperature than soluble tubulin, but reverts on solubilisation. Soluble tubulin in high sucrose shows a similar effect which is explained as due to preferential hydration preventing the protein unfolding (Lee et al., Ann. N. Y. Acad. Sci., 253, 284; 1975). Such an explanation for the membraneassociated tubulin would imply a specific interaction between the two components. Further doubt as to the

\title{
QSO redshifts and the steady state cosmology
}

John GribBin (Nature, 257, 540; 1975 ) has ably summarised the work by one of us (P.K.D.) which appeared recently (Mon. Not. R. astr. Soc., 172, $623 ; 1975)$ on the subject of the relationship between angular diameters and redshifts in Hoyle-Fowler type models of QSOs. Gribbin's ability as a mind-reader, however, must be seriously questioned when he writes in his preamble: " . . . although Das does not mention the steady state theory, it seems a reasonable assumption that any colleague of Narlikar's who discusses possible non-cosmological contributions to the redshifts of QSOs has the concept not too far towards the back of his mind."

While discounting that any such 'unholy' thought was at the back of our minds in our discussions of the Hoyle-Fowler objects (Mon. Not. R. astr. Soc., 171, 87; 1975), we would like to take this opportunity to point out that too much importance is being given by several astronomers and science writers (New Scientist, July $3,4 ; 1975)$ to any connection between the steady state theory and the noncosmological theory of QSO redshifts.

It is true that observations indicate that strong evolutionary effects are at work if the QSO redshifts are wholly cosmological in origin. It is also true that such evolutionary effects would be against the simple steady state

identity of the colchicine binding site on membrane should disappear when it can be definitely attributed to the solubilised membrane tubulin.

A role suggested for membraneassociated tubulin is as an anchor for cell surface receptors (Berlin et al., Nature, 247, 45; 1975; Edelman et al., Proc. natn. acad. Sci. U.S.A., 70, 1442; 1973). Whether it does this in its unpolymerised form or as microtubules must be investigated. Then there are the other three fibrous protein, actin, myosin and spectrin, claiming association with membranes. How all these proteins function remains an exciting and intriguing question for the future.

\section{Lasers and the Lamb shift}

\section{from Peter Knight}

THE investigation of atomic structure by conventional spectroscopic techniques (for example, excitation using electronimpact in a discharge tube and resolution hypothesis. Thus a theory of noncosmological origin of QSO redshifts is probably necessary for the survival of the steady state theory. But here the conection betwen the two ends.

For example, even if the QSOs turn out to have an appreciable noncosmological component in their redshifts, this does not validate the steady state theory which still faces other observational hurdles like the microwave background. Nor does the disproof of a particular non-cosmological redshift hypothesis in a single QSO - for example, the gravitational redshift (Wampler, et al., Astrophys. J., $198,149 ; 1975)$ in the case of $3 \mathrm{C} 48$ - particularly embarrass the steady state theory.

Indeed, regardless of any cosmological prejudices it is pertinent to ask: "Is the redshift of a QSO due to the expansion of the Universe alone?" A fair assessment of the present data does not permit an unequivocal affirmative answer to this question. Even the overall problem of the structure of a QSO still remains unsolved. Under these circumstances even theoreticians sympathetic to the steady state cosmology may work on models of non-cosmological redshifts for reasons not primarily concerned with defending that cosmology.

$$
\begin{aligned}
& \text { J. V. NARLIKAR } \\
& \text { P. A. DAS }
\end{aligned}
$$

of the emitted radiation with interferometers) is severely limited by Doppler broadening and consequently relies heavily on a precise understanding of the lineshape of the emitted radiation. To see the subtle effects of current interest within Doppler broadened spectral lines, with widths typically of the order of $\mathrm{GHz}$, the spectroscopist is forced to the limits of available technology. This is especially true of that most "fundamental" of atoms, the hydrogen atom, which, being the lightest atom suffers most from Doppler broadening. The arrival of the tunable laser in atomic physics has, however, rotally revolutionised optical spectroscopy. This long-awaited revolution is perhaps best characterised by a series of beautiful experiments on atomic hydrogen using dye laser excitation performed by T. H. Hänsch and coworkers at Stanford. In a recent paper (Phys. Rev. Lett., 35, 1262-1266; 1975) Hänsch, Lee and Wallenstein report measurements of the isotope shift of the $1 s-2 s$ transition in atomic hydrogen and deuterium, and the Lamb shift of the 1s ground state of 